\title{
Yao Language
}

National Cancer Institute

\section{Source}

National Cancer Institute. Yao Language. NCI Thesaurus. Code C154201.

A Niger-Congo Bantu language spoken mainly in Malawi, Tanzania, and Mozambique. 